\title{
Nonlinear effects in coplanar GaAs/InGaAs strained-layer superlattice directional couplers
}

\author{
Utpal Das, Yi Chen, and Pallab Bhattacharya \\ Solid-State Electronics Laboratory, Deparment of Electrical Engineering and Computer Science, The \\ University of Michigan, Ann Arbor, Michigan $48109-2122$
}

(Received 10 August 1987; accepted for publication 21 September 1987)

\begin{abstract}
We report on the performance characteristics of InGaAs/GaAs strained-layer superlattice coplanar ridge-type directional couplers realized by molecular beam epicaxy. The measured power transfer characteristics with 1.15 m incident photoexcitation demonstrate noninear couping due to absorption associated with the tails of the excitonic resonances in the quantum wells. From a theoretical fit of the measured data, the nowhear refractive index coefficient, $n_{2}$, of the multiquantum well is found to be $2.25 \times 10^{-7} \mathrm{~cm}^{2} / W$. This agrees very well with a value of $n_{2}=1.9 \times 10^{-7} \mathrm{~cm}^{2} / \mathrm{W}$ obtained independently on the same material from interferometric measurements.
\end{abstract}

Optical directional couplers are important elements in optoelectronic integrated circuits (OEIC's) using a guidedwave scheme. Waveguide couplers can serve as swiching elements. ${ }^{1,2}$ In this letter we report the coupling characteristics of coplanar GaAs/InGaAs strained-layer superlattice (SES) waveguide couplers. Nonlinear coupling behavior is seen in the SLS structures. From a theoretical fit to the data, a value for the nonlinear refractive index is derived. The latter parameter has been measured in the same materiais using a Mach-Zender interferometer.

The structures were grown by molecular beam epitaxy (MBE) on GaAs substrates. The schematics of the grown layers and structures for the coplanar coupler are shown in Fig. 1. In the measured room-temperature absorption spectrum of the InGaAs/GaAs SLS, the energy position of the heavy-hole excitonic transitions is $1.1198 \mathrm{eV}$, which is $\sim 40$ meV higher than the photon energy of the experimental 1.15-pum source used in the present measurements. Singlemole waveguides were delineated by using photolithography and ion-miling techniques.

Quantitative measurements on the coupling chatacteristics were ritade with the input guide end fred by a $2-3 \mu \mathrm{m}$ collimated and focused beam from the $1.15 \% \mathrm{~m}$ He-Ne laser. The incident light power was estimated to be $750-800 \mu \mathrm{W}$ ard its plane of polarization was TE-kike, i.e., paraliel to the layers of the heterostructures. The output from the incident and coupled guides for various coupling lengths was neasured by a liquid- $\mathrm{N}_{2}$-cooled Ge photodetector and viewed on a TV monitor via an infrared camera. The power distribution in the input and coupled guides of the coplanar structure for various coupling lengths is shown in Fig. 2 . It is interesting to note that, contrary to the expected behavior of the linear couplers, lesser power is transferred to the coupled guide for small coupling lengths. We believe this is due to the nonlinear backcoupling or switching of power from the coupled to the input guide for these short couphing lengths resulting principally from a local change of the refractive index $^{3-5}$ We estimate that a refractive index change of $\sim 0.01$ in the SLS is required to produce this effect and that it is easily achieved with the input power density available in the experimests.
In order to verify the nonlinear effect, we have calculated the couphing characteristics taking into account lossy guides coupled by a nonlinear medim. The intensity-dependert absorption coeficient and refractive index are given by

$$
\begin{aligned}
& \alpha(D)=\alpha_{0}+\alpha_{2} l \\
& n(D)=n_{0}+n_{2} \bar{l}
\end{aligned}
$$

where $\alpha$ and $p$ are the absorption coefficient and refractive index of the ronlinear medium. Screening of the Coulonb interactions and band flling effects under high-excitation levels gives rise to a quenching of the exciton resonance and a change in $\alpha$ or $n$. For switching applications, where transient conditions are operative, $\alpha$ can be obtained from a solution of the rate equation for excess carriers generated by photoexcitation, as outhed by Wherret. ${ }^{5}$

\section{One can express}

$n_{2}=F o$,

and this dependence can be nonlinear for large $\alpha$, as experimentally found by Li Kam Wa et $a l^{3}$ The coupled-mode equations for the propagation of power in lossy nominear directional couplers are then

$$
\begin{aligned}
& \frac{d a_{1}}{d z}=i \beta a_{1}-\frac{\alpha}{2} a_{1}+i C a_{2}-i \alpha F\left|a_{1}\right|^{2} a_{1} \\
& \frac{d a_{2}}{d z}=i \beta a_{2}-\frac{\alpha}{2} a_{2}+i C a_{1}-i \alpha F\left|a_{2}\right|^{2} a_{2}
\end{aligned}
$$

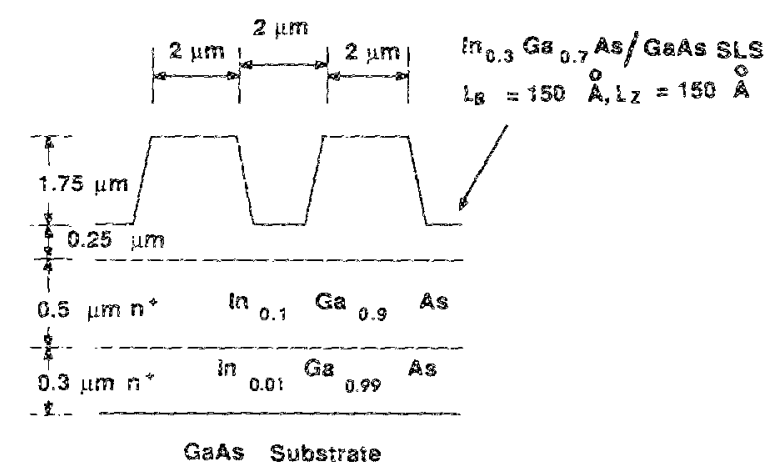

MG. 1. Structure of coplanar waveguide coupler with $\operatorname{In}_{0.3} \mathrm{Ga}_{0.7}$ As/CuAs strained-layer superiatice guiding and coupling regions realized by molecular beam epitaxy. 


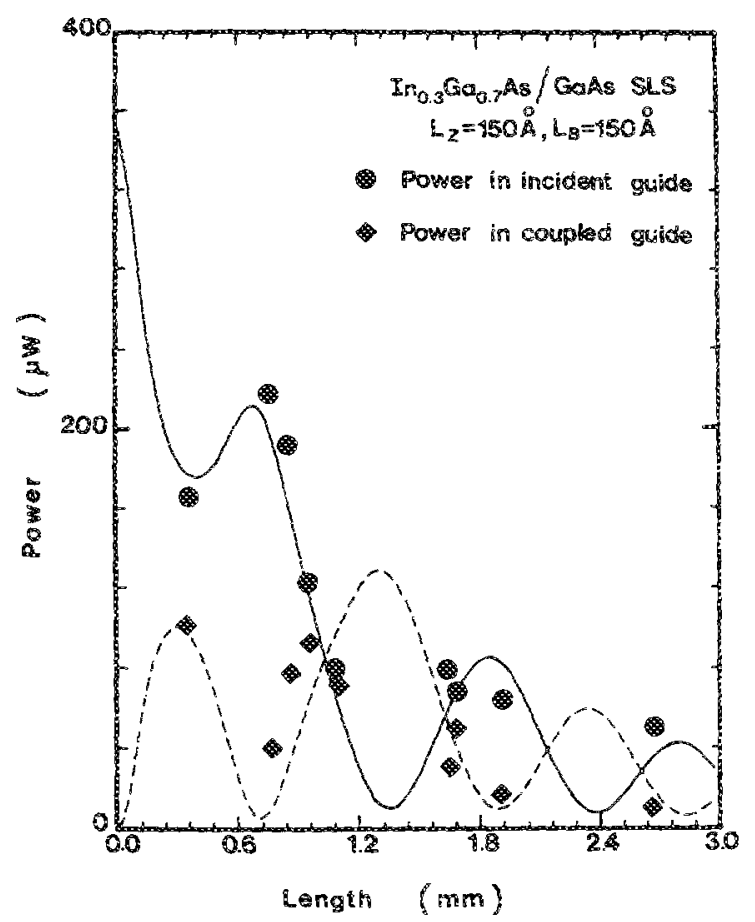

HKG. 2. Power transfer characteristics of the coplanar strained-layer superlattice (SLS) waveguide coupler. The data for differont coupling lengths were obtained by the cutback method. The solid and dashed curves are culculated data for the input and coupled guides, respectively.

where $a_{1}$ and $a_{2}$ are the amplitudes of the two waves, expressed as

$$
\begin{aligned}
& a_{1}=A_{1} e^{-(\alpha / 2) z+i k z}, \\
& a_{2}=A_{2} e^{\cdots(\alpha / 2) z+i k z},
\end{aligned}
$$

and $C=\pi / 2 L_{C}$, where $E_{C}$ is the critical coupling length (100\% power transfer).

Equations (3a) and (3b) have been numerically solved using the Runge-Kutta technique, to the experimental data. The solid and dashed lines in Fig. 2 are the calculated powers in the two guides obtained for $n_{2}=2.25 \times 10^{-7} \mathrm{~cm}^{2} /$ $W, a=30 \mathrm{~cm}^{-1}$, and $L_{C}=0.4 \mathrm{~mm}$.

Single waveguides typically $1.1 \mathrm{~mm}$ long and of the same dimensions as the coupier were fabricated with the SLS material. Aach-Zender interferometer was constructed with the guide placed in one arm, as shown in Fig. 3. The 1.15-14m light in this path was guided in the SLS region. The interference fringes observed due to the interference effect are shown in the inset of Fig. 4. A shift of the fringes was observed and recorded for increasing light intensity. The corresponding phase shifts were also calculated. The variation of the phase shift with input power is shown in Fig. 4. Assuming a linear dependence of the phase shift on input powe: one can express the nonlinear refractive index in the SLS as

$$
n_{2}=\frac{\Delta \phi}{P_{i}}\left[\frac{2 \pi}{\lambda_{0} A}\left(\frac{1-\exp \left(-\alpha l_{2}\right)}{\alpha}\right)\right]^{-1},
$$

where $\Delta \phi$ is the relative phase shift, $P_{i}$ is the inpus power, $A$ is the guide cross section, $l_{z}$ is the length of the guide, and $\lambda_{0}$ is the free-space wavelength of the incident light. Using $\mathbf{E q}$.

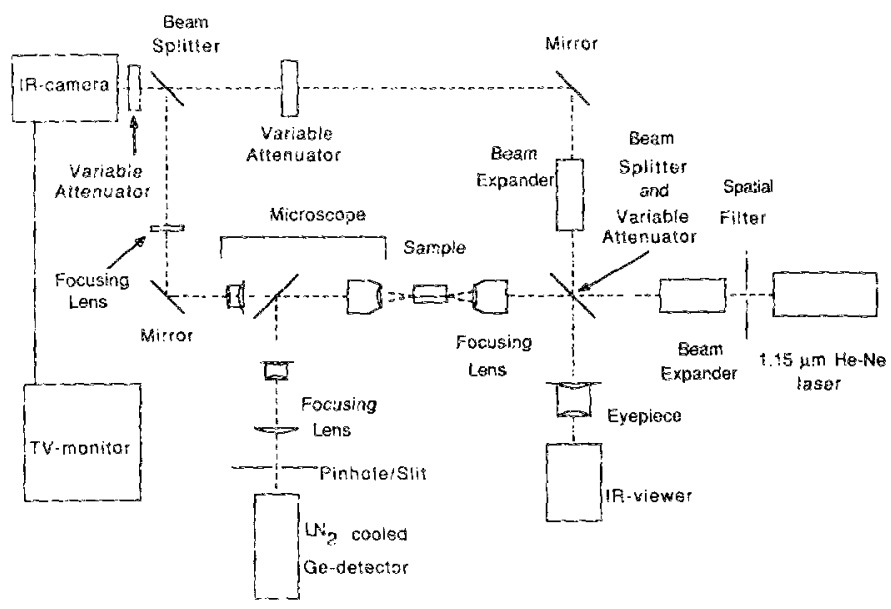

FIG. 3. Scinematic of the Mach-Zender interferometer for measurement of nonlinear refractive index. A $25-\mu \mathrm{m}$ pin hole was used to detect the output light.

(5) for the data in Fig. 4, a value of $n_{2}=1.9 \times 10^{-7} \mathrm{~cm}^{2} / \mathrm{W}$ is derived, which is in good agreement with that obtained from the coupled power data.

A comment should also be made here about the possible refractive index change due to thermal effects. It has been recently established by $\mathrm{L} \mathrm{K}$ am Wa al ${ }^{3}{ }^{3}$ that thermal heating of similar GaAs/AlGaAs multiple quantum well (MQW) couplers essentially moves the fringes in the opposite direction and results in a positive nonlinear refractive index coefficient. This sign of change would not show the nonlinear coupling observed by $\mathrm{L} i \mathrm{Kam} W \mathrm{~W}$ et $a l^{3}$ or by us. It may, therefore, be concluded that the noninearity observed by us is predominantly due to high-speed nonlinear excitonic effects in the $\mathrm{MQW}$.

To our knowledge this is the first demonstration of noninear coupling behavior in SLS material. The effect can be

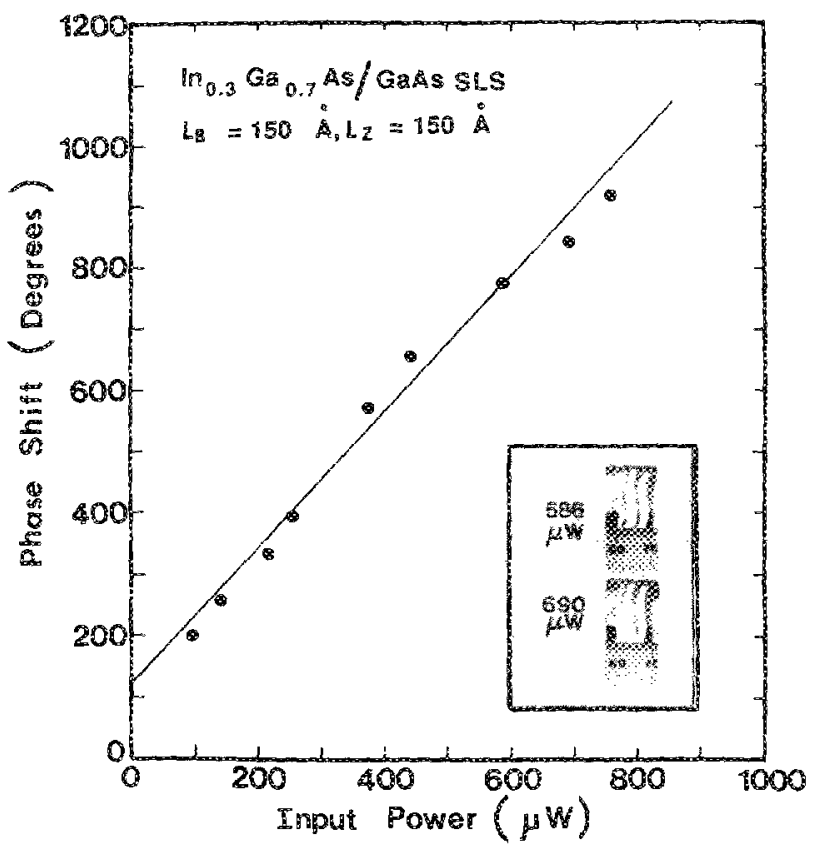

FIG. 4. Measured variation of phase shift with power incident on the SLS guide in one arm of a Mach-Zender interferometer. The inset shows the observed fringes at the output for (a) $586 \mu \mathrm{W}$ and (b) $690 \mu \mathrm{W}$. 
seen more clearly, and utilized for photonic switching with higher light intensities, and these experiments are in progress in our laboratories.

The authors wish to thank Paul R. Berger for growing the structures and for his help in preparing the samples. Discussions with $G$. Veztoli are gratefuly acknowledged. The work was supported by the National Aeronautical and Space Administration under gran NAG-1-555 and the Lightwave Fechnology Program, National Science Foundation under grant ECE-8610803.
'T. M. Benson, T. Murotani, P. N. Robson, and P. A. Houston, IEEE Trans. Electron Devices E19, 1477 (1982).

2h. D. Westbrook, B. N. Kebson, and A. Majerfeld, Electron. Lett. 15, 99 (1979).

${ }^{3}$ P. Li Kam Wa, J. E. Sitch, M. J. Mason, J. S. Roberts, and P. N. Robson, Electron. Lett. 21,26 (1985).

${ }^{4}$ M. Cada, R. C. Gauthier, M. E. Paton, and J. Chrostowski, Appl. Phys. Lett. 49,755 (1986).

${ }^{5}$ G. 1. Stegeman, C. T. Seator, C. N. Ironside, T. Cullen, and A. C. Walker, Appl. Phys. Leti. 50, 1035 (1987).

'B. S. Wherrett, in Nonlinear Optics: Materials and Devices, edited by $\mathbb{C}$. Flytzanis and J. L. Ondar (Springer, Berlin, 1986), pp. 180-221. 